\title{
Analysis of microstrip antennas with inhomogeneous and finite-sized substrate
}

Yi-Hsin Pang* and Ruey-Beei Wu Department of Electrical Engineering National Taiwan University, Taipei, Taiwan, R.O.C E-mail: d85014@ew.ee.ntu.edu.tw,rbwu@ew.ee.ntu.edu.tw

\begin{abstract}
Microstrip antennas with inhomogeneous, finite-sized substrate and ground plane are studied. Applying the finite element-boundary integral (FE-BI) method, the electric and magnetic fields radiation on the dielectric boundary of the finite microstrip structures can be solved numerically, without the assumption of infinite substrate. The numerical result is compared with the one obtained by integral equation method for the case of homogeneous substrate, and shows good consistence with each other. The effects of inhomogeneous substrate are also studied.
\end{abstract}

\section{INTRODUCTION}

Microstrip antennas are widely used, since they are lightweight, conformal and easy to fabricate. Analyses of microstrip antennas are also available in extensive literature. Integral equation utilized Sommerfeld formulation is one of the popular full-wave analysis methods, such as [1]. An important assumption of this method is that the substrate and ground plane are of infinite extent in the transverse direction. Without this assumption, the associated Green's functions are not available and the method fails. This assumption is not practical, however, especially for the application of personal wireless communications. The diffraction due to the presence of the edges of substrate and ground plane would affect the various characteristics of antennas, such as the resonant frequency[2], input impedance[3], and radiation patterns[4]. Several methods have been proposed in the literature to deal with these problems, such as integral equation method[5][6] and the method of lines[7]. In this study, we adopt the finite-element boundary-integral (FE-BI) method[8] since it is more flexible for arbitrarily shaped, inhomogeneous, and finite-sized substrates

\section{OUTLINE OF THE THEORY}

Fig. 1 depicts the problem of interest, a microstrip antenna with finite-sized substrate and ground plane. Denote the boundary of the whole antenna structure as surface $S$,

This work was supported in part by the National Science Council, Republic of China, under Grant NSC 88-2213-E-002-074, and the Ministry of Education under Grant 89-E-FA06-2-4. 
inside which a substrate with relative permittivity $\varepsilon_{r}(\mathbf{r})$ is assumed. $\overrightarrow{\mathbf{J}}^{i}$ and $\overline{\mathbf{M}}^{i}$ represent the feeding sources for the antenna. Outside $S$ is free space where the antenna radiates. Another assumption imposed is that metals are of zero thickness and, although not necessary, are perfectly electrical conductors (PEC). The original problem is divided into two equivalent problems, as shown in Fig. 2. Inside $S$, finite element method is applied for the inhomogeneous region. For the exterior problem, equivalence principle is applied and the fields outside $\mathrm{S}$ can be expressed in terms of the equivalent surface current $\left(\vec{J}^{\prime}, \vec{M}^{s}\right)$ on the boundary S[9]. Matching the boundary conditions on S, the magnetic field integral equation (MFIE) for the unknown equivalent surface currents can be obtained. Note that the boundary fields $(\overrightarrow{\mathbf{E}}, \overrightarrow{\mathbf{H}})$ in FEM are not independent of the equivalent surface current $\left(\overrightarrow{\mathbf{J}}^{\mathbf{x}}, \overrightarrow{\mathbf{M}}^{\mathbf{3}}\right)$ in the equivalent exterior problem. In fact, they are related as $\overrightarrow{\mathbf{E}} \times \hat{\mathbf{n}}=\overrightarrow{\mathbf{M}}^{s}$ and $\hat{\mathbf{n}} \times \overrightarrow{\mathbf{H}}=\overrightarrow{\mathbf{J}}^{s}$, where $\hat{\mathbf{n}}$ is the unit outer normal vector for the boundary $\mathrm{S}$.

Expanding the unknown electric fields with edge-based tetrahedral Whitney elements and applying the Ritz procedure, the variational equation for the interior problem can be cast into a matrix equation with the boundary and feeding electric fields unsolved. Expanding the unknown equivalent surface electric currents with 2D Whitney elements and the magnetic currents with 2D Rao-Wilton-Glisson (RWG) functions[10], and applying the method of moments with RWG functions as the weighting functions, the MFIE can be also cast into a matrix form. These two matrix equations are then solved numerically by Biconjugate gradient method (Bi-CGM) and the unknown fields and currents are obtained.

\section{RESULTS}

Take the rectangular microstrip antenna shown in Fig. 3(a) as the first example. In this case, the size of ground plane equals to the size of the patch antenna. It was solved by integral method (IE) described in [5] and by the FE-BI method here. As indicated in Fig. 3(b), the resonant frequencies obtained by these two methods differ each other by only $0.7 \%$, and shows good consistency with each other.

Consider an air-filled rectangular cavity inside a microstrip antenna with a finitesized substrate. As indicated in Fig. 4(a), the size of patch is $30 \mathrm{~mm} \times 20 \mathrm{~mm}$, the size of substrate is $42 \mathrm{~mm} \times 32 \mathrm{~mm} \times 1.5 \mathrm{~mm}$, and the cavity is of size $6 \mathrm{~mm} \times 20 \mathrm{~mm} \times 1.5 \mathrm{~mm}$. This problem is difficult to be solved by IE method because of the inhomogeneous substrate. However, it is much easier for FE-BI method. The distance between the cavity and the edge of substrate is denoted as $\mathrm{L}$. The resonant frequency and $\mathrm{Q}$ factor oscillate as $L$ varies and are presented in Fig. $4(\mathrm{~b})$. The dashed line indicates the values for which 
the substrate is homogeneous with dielectric constant 4.55 and no cavity exists. When the cavity moves to the edge of the patch where the magnitude of electric field is the largest, the resonant frequency reaches its maximum. It is because that the effective dielectric constant becomes smaller. Also, the $Q$ factor has its minimum value because of the smaller stored energy.

\section{SUMMARY}

In this paper, microstrip antennas with inhomogeneous substrate of finite extent are analyzed by FE-BI method. It can also be applied to discuss the finite dielectric effects on the radiation characteristics of other printed antennas such as the $\mathrm{CPW}$-fed microstrip antennas. Besides, structures of arbitrarily shaped substrate deserve further study.

\section{REFERENCE}

[1] J. R. Mosig, "Arbitrarily shaped microstrip structures and their analysis with a mixed potential integral equation," IEEE Trans. Microwave Theory Tech., vol. 36, pp. 314-323, Feb. 1988.

[2] M. Gimersky and J. Bornemann, "A modified method-of-moments technique for the full-wave analysis of imperfect conductors on lossy and finite-extent substrates," IEEE MTT-S Digest, pp. 715-718, 1996.

[3] H. Iwasaki, "A back-to-back rectangular-patch antenna fed by a CPW" IEEE Trans. Antennas Propagat., vol. 46, no. 10, pp. 1527-1530, Oct. 1998.

[4] J. Huang, "The finite ground plane effect on the microstrip antenna radiation patterns," IEEE Trans. Antennas Propagat., vol. 31, no. 4, pp. 649-653, July 1983.

[5] T. K. Sarkar, S. M. Rao, and A. R. Djordjevic, "Electromagnetic scattering and radiation from finite microstrip structures," IEEE Trans. Microwave Theory Tech., vol. 38 , no. 11, pp. 1568-1575, Nov. 1990.

[6] S. A. Bokhari, J. R. Mosig, and F. E. Gardiol, "Radiation pattern computation of microstrip antennas on finite size ground planes," IEE Proc.-H, vol. 139, No. 3, pp. 278-286, June 1992.

[7] M. Drissi, P. Herve, and J. Citerne, "A rigorous analysis of planar antennas having a finite size substrate," IEEE Int. Symp. Dig. Antennas Propagat., pp. 1462-1465, 2000.

[8] J. L. Volakis, A. Chatterjee, and L. C. Kempel, Finite element method for electromagnetics, IEEE Press, Chap. 7, 1998.

[9] R. F. Harrington, Time-harmonic electromagnetic fields, McGraw-Hill, 1993.

[10] S. M. Rao, D. R. Wilton, and A. W. Glisson, "Electromagnetic scattering by surfaces of arbitrary shape," IEEE Trans. Antennas Propagat, vol. 30, no. 5, pp. 409-418, May 1982 


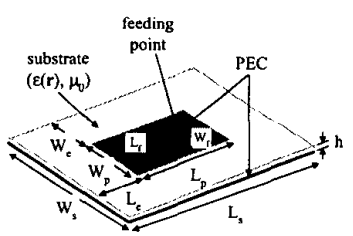

Fig. 1. A microstrip antenna with finite-sized substrate and ground plane.

(E)

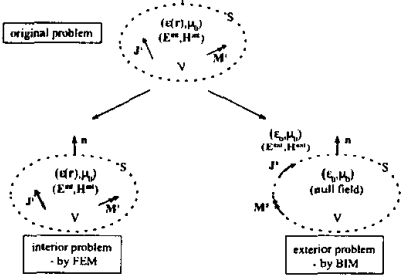

Fig. 2. The equivalent problems for FE-BI method.

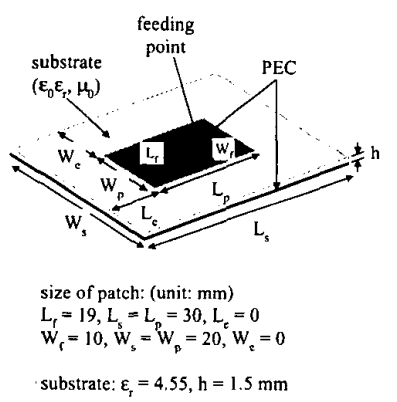

(a)

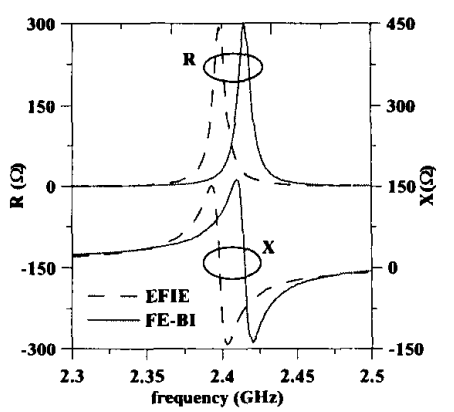

(b)

Fig. 3. A rectangular microstrip antenna with equal-sized substrate and ground plane (a) structure, (b) input impedance.
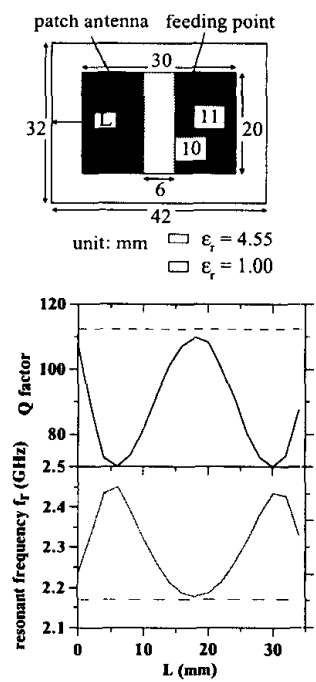

Fig. 4. An air-filled rectangular cavity inside a microstrip antenna with finite-sized substrate and ground plane (a) structure, (b) resonant frequency and $Q$ factor. 